\title{
Estudio Exploratorio sobre una Escala de Autoevaluación para la Depresión en Niños y Adolescentes
}

\author{
Dra. Eliana Alvarez M. ${ }^{1}$; Dr. Humberto Guajardo S. ${ }^{2}$; Dr. Ricardo Messen H. ${ }^{3}$. \\ Aplication of a Self-Rating Scale in the Diagnosis of \\ Depressive Disorder in Children and Adolescents
}

\begin{abstract}
Birleson's self-rating for the evaluation of depressive disorders in children and adolescents was aplied to 8 patients with confirmed depressive disease, 92 patients with other Kinds of psychiatric symptoms and 135 normal school children and adolescents matched by age and sex. The mean scores obtained by the psychiatric patiens as a whole $(\mathrm{n}=100$, score $=13.10)$ were not statisfically different from those of healthy controls $(12.50)$, but they were significantly higher in depressive patients $(n=8$, score $=22)$ in comparisson with other psychiatric patients $(n=92$, score $=12.46(\mathrm{p}<0.01))$.

(Key words: Childhood depressive disorder, Diagnosis. Self-rating scale for depressive disease).
\end{abstract}

Durante mucho tiempo la Depresión Infantil ha sido objeto de controversias existiendo opiniones que afirman y otras que rechazan su existencia como un Síndrome Clínico diferente $1,2,3$.

Las principales dificultades en la individualiza. ción de esta afección se ubican en el campo del điagnóstico ya que éste se realiza en base a criterios muy diversos. Esta disparidad da como resultado el que se cataloga como depresivos a un grupo muy heterogéneo de niños, lo que hace casi imposible comparar diferentes poblaciones de pacientes y efectividad de los tratamientos.

1. Psiquiatra. Departamento de Salud Mental Sur. Facultad de Medicina. Universidad de Chile.

2. Psiquiatra. Jefe del Servicio de Psiquiatría Infantil. Hospital Exequiel González Cortés.

3. Psiquiatra. Servicio de Psiquiatría Infantil. Hospitạl Exequiel González Cortés.
En nuestra práctica clínica diaria tenemos la impresión de encontrarnos cada vez más con nínos que pueden considerarse como portadores de un trastorno depresivo, sin embargo en revisio. nes estadisticas preliminares en nuestro Servicio encontramos cifras de depresión que no pasaban del $1 \%$ del total de consultas, lo que contrasta con lo anteriormente expuesto.

E] creciente interés por la validación del trastorno depresivo de la infancia ha llevado a intentar unificar los criterios de diagnóstico clinico y es así como diferentes grupos de investigadores han hecho diversas proposiciones.

Dentro de los más importantes mencionaremos los de Weimberg (el primero), R.D.C. (Research Diagnostic Criterial) D.S.M. III (Manual diagnóstico y estadistico III), etc. Es un hecho actualmente establecido que los criterios que se han utilizado en el điagnóstico de depre. sión mayor del adulto son válidos en los niños ${ }^{4}$. 
Por otra parte se han desarrollado escalas para medir los estados de ánimo tanto en adultos como en niños CDI (Children Depresion Inventory) K-SADS, ISC (Kovacs) BDI (Beck). Algunos de ellos presentan cierto nivel de complejidad, son demasiado sensitivos, o bien muy extensos para ser usados en forma habitual en nuestra realidad clínica ${ }^{5}$.

En 1982, Birleson desarrolló su escala de autoevaluación para la depresión, una de las últimas en publicarse ${ }^{6}$.

Por su fácil uso y los resultados descritos nos interesamos por probarla en nuestro medio, estudiando su utilidad y su correlación con la clínica, en los pacientes que consultan en el Servicio de Salud Mental Infantil del Hospital Exequiel González Cortés.

\section{MATERIAL Y METODO}

El Hospital Exequiel González Cortés atiende Ia población del Sector Sur de Santiago correspondiente a las comunas de San Miguel, La Cisterna, San Bernardo y Buin.

Los pacientes son referidos por los Pediatras de los Consultorios periféricos ya sea porque pesquisan ellos los problemas de Salud Mental o bien a solicitud de los padres y/o profesores.

La escala de depresión fue aplicada a un grupo clínico y a un grupo control.

El grupo clínico estuvo constituido por 100 casos que consultaron en nuestro Servició excluyéndose aquellos que presentaban Retardo Mental o Psicosis. Las edades fluctuaron entre 9 y 18 años; 44 pacientes eran mujeres y 56 hombres.

E] grupo control consistió en 135 alumnos de Colegios de Enseñanza Básica y Media con una distribución similat por sexo y edad que el grupo ae pacientes.

El grupo clínico recibió ademas una entrevista clínica habitual, realizada por Psiquiatras y/o Psicólogos, a los cuales previamente se les entregó los criterios clínicos para el diagnóstico de depresión (Tabla 1). Estos fueron los mismos que usó Birleson en su trabajo original, quien realizó pruebas de confiabilidad y acuerdo entre distin. tos entrevistadores Ilegando a cifras cercanas al $100 \%$.

Los investigadores aplicaron el cuestionario para la depresión .(apéndice) en el grupo de pacientes y grupo control. En el grupo clínico desconocian el diagnóstico realizado por los médicos o psicólogos. El cuestionario consta de 18 items que deben ser respondidos por el entrevistado eligiendo una de tres alternatjvas.

Al contrario, si se comparan los resultados
Tabla 1

\section{Criterios Clínicos para el Diagnóstico de Depresión (Birleson, 1981)}

I. Evidencia de infelicidad, tristeza, miseria, labilidad emocional (tendencia al llanto), que se haya expresado recientemente

II. Historia de cambio conductual que haya durado pot lo menos 2 semanas, pero menos đe un año.

III. Evidencia de dificultad reciente a las relaciones sociales y/o disminución del rendimiento escolar.

IV. Presencia de 2 o más de los siguientes sintomas:

- Trastorno del sueño

- Trastorno del apetito

- Pérdida đe energía o interés habitual

- Reducción de la actividad

- Expresión de ideas autodepreciatorias

- Amenazas o conductas suicidas

- Irritabilidad creciente

- Quejas somáticos nuevos

- Conducta de vagancia

- Delirio y/o alucinaciones depresivos

Para hacer el diagnóstico deben cumplirse los cuatro criterios

obtenidos por los pacientes depresivos con el resto de los pacientes de la muestra clínica y por el grupo control se observa que los 8 depresivos alcanzan un puntaje promedio de 22 , mientras que en los restantes 92 los pacientes es de 12,46 y 12,5 en el grupo control de 135 sujetos.

Las diferencias observadas son estadisticamente significativas, lográndose una estrecha correlación entre puntaje elevado y diagnóstico de depresión.

La sensibilidad de la prueba (No de depresivos con puntaje alto) fue de $87,5 \%$ y su especificidad ( $N^{\circ}$ de no depresivos con puntaje bajo) de $93 \%$. El valor predictivo fue de $50 \%$.

\section{COMENTARIO}

Es importante destacar, en primer lugar que al incorporar criterios para el diagnóstico de depresión aumenta la frecuencia con que se efectúa ese diagnóstico, desde $1 \%$, cifra tradicional en nues. tro Servicio, a 8\% (Tabla 2).

Esto apoya la impresión de que los trastornos depresivos de la infancia son sub-diagnosticados, hecho que debe ser corregido ya que se sabe que en el campo de la Psiquiatria Infantil no es indiferente no hacer el diagnóstico de una patología, o bien hacetlo tardíamente.

Esta cifra de $8 \%$ es aún menor que la encontrada por autores extranjeros, que flutúa entre un 
Tabla 2

Distribución de las causas de Consulta en 100 Pacientes (9 - 18 años)

\begin{tabular}{lr}
\hline - Síndrome Déftcit de Atención & 27 \\
- Trastornos emocionales & 24 \\
- Trastornos del sueño & 8 \\
- Trastornos del aprendizaje & 7 \\
- Trastornos de conducta & 7 \\
- Epilepsia & 3 \\
- Uso de drogas & 12 \\
- Otros (Trast. del comer, cefaleas, & 8 \\
- trastornos psicomáticos, etc.) & \\
\hline
\end{tabular}

100

10 a $27 \%$ en nuestra clinica 4 . Esto puede indicar que se necesita más la búsqueda de pacientes, 0 bien que las caracteristicas psicopatológicas de nuestra población son diferentes, con una incidencia realmente menor de depresión. Se necesita más investigación para responder a esta interrogante.

A cada item se le otorgó una calificación de 0 -1 - 2 en el sentido de la alteración.

\section{RESULTADOS}

Al examinar los diagnósticos efectuados en la muestra de 100 pacientes (Tabla 2) se obset va que el diagnóstico de depresión se realizó en 8 casos, 7 de los cuales correspondieron al sexo Femenino y 1 al Masculino.

Los diagnósticos más frecuentes fueron síndrome de Déficit de Atención (27 casos) y trastornos emocionales (24 casos), que son los casos que mayoritariamente consultan en nuestro servicio. No aparecen el Retardo Mental y la Psicosis, ya que fueron excluidos de ta muestra.

Al analizar los resultados obtenidos en el cuestionatio (Tabla 3) por el grupo clínico y el grupo control se abserva que no existen diferen. cias estadisticamente significativas, siendo el puntaje promedio del grupo de pacientes 13.10 y los controles 12.50. Tampoco hubo diferencias significativas entre los puntajes obtenidos por hombre $y$ mujeres, en ninguno de los dos grupos.

El hecho de que no exista diferencia significativa entre las calificaciones promedio del grupo de pacientes y el grupo control se esperaba, por cuanto sólo los pacientes depresivos debieran tener notaciones elevadas en el cuestionario. Esto se observa sólo cuando se comparó los depresivos
Tabla 3

Calificicación Obtenida en la Escala de Depresión en Pacientes y Grupo Control

\begin{tabular}{lrrrrr}
\hline & \multicolumn{2}{c}{ Controles } & & \multicolumn{2}{c}{ Pacientes } \\
\cline { 2 - 3 } \cline { 5 - 6 } & \multicolumn{1}{c}{ No $^{\circ}$} & Promedio & & No & Promedio \\
\hline Mujeres & 78 & 13,78 & & 44 & 15,27 \\
Hombres & 57 & 10,73 & & 56 & 11,44 \\
Total & 135 & 12,50 & & 100 & 13,10
\end{tabular}

Las diferencias obtenidas no son significativas

con el resto de Jos pacientes y con el grupo control.

En este sentido la escala demostró ser muy útil por cuanto discriminó no sólo entre los depresivos y controles nórmales, sino también entre pacientes con depresión y otro tipo de afección psiquiátrica. Tampoco se observó diferencia significativa por sexo ni en el grupo total de pacientes ni con los controles. Llamó sin embargo la atención que las mujeres (pacientes y controles) tenían calificaciones numéricas más altas que los varones de los mismos grupos, valores que sin ser estadísticamente significativos, indican que la variable sexo deberá ser considerada como. importante de controlar en futuros estudios tanto más si se considera que de $\operatorname{los} 8$ depresivos, 7 fueron mujeres.

El $87,5 \%$ de los pacientes depresivos fue diag. nosticado como tal por el cuestionario, es decir, obtuvo calificaciones elevadas. El $93 \%$ de los pacientes no depresivos tuvo calificaciones bajas, bo que nos permite afirmar que la sensibilidad y especificidad de la escala son bastante altas igual o mayor que otras pruebas utilizadas en Psiquiatría y en Medicina General.

El valor predictivo de un $50 \%$ tambien es comparable a otras pruebas y exámenes, pero pensamos que puede mejorar en futuros estudios que amplíen el número de casos en el grupo depresivo.

Si se comparan nuestros resultados con el trabajo original de Birleson resaltan algunas diferencias. En lnglaterra la escala se aplicó en niños desde los 7 años de edad lo que no fue posible en nuestro caso, porque la población beneficiaria, en su gran mayoría, tuvo serias dificultades para leer y comprender el texto antes de los 9 años de edad, que por esta razón se tomó como limite inferior.

Los valores obtenidos por Birleson son en general más bajos que los nuestros (4 puntos promedio para niños normales, 8 puntos para pacientes y 17 para los depresivos). Estas diferencias pueden estar influidas por variables de tipo 
socio-cultural, dado que nuestros niños corresponden a un sector de bajos ingresos, con estándar de vida inferior al inglés. Pensamos que en el futuro será importante aplicar esta escala en estratos socio-económicos medio y alto.

En el trabajo original se propuso utilizar una calificación igual o superior a 13 pata considerar la prueba como positiva, con valores de sensibilidad de $80 \%$.

En nuestra muestra, utilizando una calificación de 19, la especificidad y la sensibilidad resultaron cercanas a $90 \%$.

Finalmente, a partir de lo que hemos expuesto podlemos afirmar que el sólo hecho de incorporar criterios objetivos aumenta la frecuencia con que se realiza el diagnóstico de depresión. Este proce. so puede ser aún más preciso si a lo anterior se agrega la ayuda de una escala que permita medir de alguna manera los estados de ánimo. La escala de depresión, mostró una alta correlación entre sus resultados y el diagnóstico clínico de esta afección.

Si bien es cierto que se necesita más investigación es evidente que disponer de un instrumento como este puede tener muchas aplicaciones. Por ejemplo sería de gran valor utilizarlo como prueba de rastreo en poblaciones de niños en general y en niños con riesgo de depresión (institucionalizados, hospitalizados, hijos de padres depresivos, etc.). Otra ventaja adicional es que para estos efectos y dado su fácil uso, puede ser usado no sólo por psiquiatras sino también por personal no especialista.

Por último, y no menos importante, en el campo psiquiátrico también sería de mucha utilidad contar con una medida objetiva de depresión

Apéndice

Cuestionario

SIEMPRE A VECES NUNCA

1. Pienso que los días que vienen serán agradables.

2. Siento ganas de llorar.

3. Tengo ganas de arrancar o escapar.

4. Tengo dolores de estómago.

5. Quiero salir a jugar fuera de casa.

6. Duermo muy bien.

7. Tengo mucha energía.

8. Disfruto mis comidas.

9. Puedo arreglármelas solo, valerme por mí mismo.

10. Creo que no vale la pena vivir.

11. Me resultan bien las cosas que hago.

12. Disfruto lo que hago igual que antes.

13. Me gusta hablar con mi familia.

14. Tengo sueños horribles.

15. Me siento muy sólo.

16. Me animo fácilmente, me entusiasmo con mucha facilidad.

17. Me siento tan triste que difícilmente lo soporto.

18. Me siento muy aburrido, lateado, choreado.

\section{INSTRUCCIONES:} pasada

Por favor, conteste tan honestamente como puedas; las frases se refieren a como tú te has sentido la semana

No hay respuestas correctas, es importante que cuentes cómo te has sentido. 
ya no sólo en el diagnóstico sino también en la evolución de la psicoterapia o de los tratamientos con medicamentos o cualquier método que se utilice en niños con problemas en el humor.

\section{RESUMEN}

Se aplicó el cuestionario para la depresión infantil de Birleson en una muestra de 100 pacientes del Servicio de Psiquiatria Infantil del Hospital Exequiel González.Cortés. El mismo instrumento se utilizó en un grupo control.

Se comprobó que los resultados del cuestionario tenian alta correlación con el diagnóstico clínico de Depresión.

\section{REFERENCIAS}

1. Rutter, M.: Relationships between Child and adult psichiatric disorders Acta psychiat. Scand 2: 3, 1971.

2. Groham $P_{\text {.: }}$ Depression in prepubertal children Dev Med. Child. NeuroL 16: 340, 1974.

3. Cantwell, D.P. y Carlson G.: A survey of depressive symtome in child and adolescent psychiatric population. J. Amer. Acad. Child. Psychiat. 18: 587, 1979

4. Puig-Antich, J. Weston B.: The diagnosis and treatment of major depressive disorder in child hood. Ann. Rev. Med. 34: 231, 19 B3.

5. Hamilton M.: Development of a rating scale for primary depressive illness. Br. J. Soc. Chin. Psychol 6: $278,1967$.

6. Birleson, P.: The validity of depressive disorder in childhood and the development of a self-rating scale a research report. J. Child Psychol and Psychiat. Vol. 22: 73,1981 . 